\title{
Why Answer this Question? Experts' Behaviors on Educational Community Question-Answering Platforms
}

\author{
Harry Stokhof ${ }^{1}$, Kalliopi Meli ${ }^{2}$, Konstantinos Lavidas ${ }^{2}$ and Dimitrios Grammenos ${ }^{3}$ \\ ${ }^{1}$ HAN University of Applied Sciences, The Netherlands \\ ${ }^{2}$ University of Patras, Greece \\ ${ }^{3}$ Institute of Computer Science, Foundation of Research and Technology, Greece \\ harry.stokhof@han.nl \\ kmeli@upatras.gr (corresponding author) \\ lavidas@upatras.gr \\ gramenos@ics.forth.gr
}

\begin{abstract}
This study explores the factors that influence experts' regular contribution to educational community questionanswering (CQA) platforms. Providing answers is essential for sharing knowledge on CQA platforms, but it also affects learners' progressive inquiry. Therefore, the purpose of this study is to develop and test a theoretical model that aims to explain which factors influence whether experts answer questions on educational CQA platforms and how these factors correlate with each other to form a "map" of experts' respective behavior. We examined experts' perceptions of three dimensions: the CQA platform's usability, the quality of questions asked, and the added value of answering these questions. We examined the factors involved in these dimensions from the perspective of the Theory of Planned Behavior to connect them with the experts' perceptions, intentions, and actions on a CQA platform. As our case study, we took the 100mentors web and mobile app, a small-scale platform that addresses learning communities around the world, and we conducted a survey for their registered experts $(N=126)$. The factorial structure indicated that experts first perceived the question quality mostly based on its relevance to their expertise or experience (question quality); secondly, that their intention to answer was mainly set by their motives to make a difference for the learner and partially by the user-friendliness of the platform (added value of answering and CQA platform's usability); and finally, that their actions were connected to the regular use of the platform for answer-sharing (CQA platform's usability). A future research challenge is to test the factorial structure in large-scale educational CQA platforms. The further confirmation of the expert behavior pattern can have a practical implication for the platforms to guide their expert community more efficiently and for the learners to pursue their learning through progressive inquiry.
\end{abstract}

Keywords: Educational platform; expert contribution; theory of planned behavior; student questioning; expert motives; structural model

\section{Introduction}

"An essential aspect of progressive inquiry is to set up questions or problems that guide the process of inquiry. Without a research question, there cannot be a genuine process of inquiry although traditional teaching often takes place without any questions whatsoever" (Hakkarainen, 2003). Fortuitously, in our digitally-networked society, learners are no longer exclusively dependent on formal education or textbooks to find the information they need, but can also ask their questions using search engines and social media. Using social media networks for learning purposes is also referred to as "social learning" (Guan, et al., 2018). Tools that are particularly useful for social learning include CQA platforms, which are rapidly increasing in both number and size (Figueroa, 2017). CQA platforms are online information repositories that develop dynamically with questions and answers posted by their users (Neshati, Fallahnejad and Beigy, 2017).

However, to efficiently accommodate learners' progressive inquiry, an increasing number of posted questions on the CQA platform is not enough: answers should be given as well, preferably by experts, namely by individuals "who are knowledgeable in a given topic" (Neshati, Fallahnejad and Beigy, 2017, p.1028). To make a CQA platform an effective source of information for learners, it is crucial that the question response rate is high. Yet, regardless of experts' degree of participation on CQA platforms, many questions remain unanswered (Asaduzzaman, et al., 2013; Liu and Jansen, 2018; Procaci, et al., 2019; Yuan, et al., 2020). Although information technology literature has extensively explored the factors that influence experts' answer-sharing on CQA platforms, there is remarkably little known about their participation on educational CQA platforms, wherein they address specific learning communities such as school classes and training programs with specific instructional goals (Le, Shah and Choi, 2017). Particularly in these cases, getting answers is essential for the development of learners' progressive inquiry (Lakkala, 2008; Stokhof, 2018). Therefore, the purpose of this study 
is to develop and test a theoretical model that aims to explain which factors influence whether experts answer questions on educational CQA platforms and how these factors correlate with each other to form a "map" of experts' respective behavior (Burel, et al., 2015; Stokhof, Meli and Lavidas, 2021). In this respect, we developed a questionnaire based on the pertinent literature review and shared it with the experts actively using the 100mentors educational platform. We used factor analysis of the questionnaire responses to validate and refine the factors that affect experts' behavior and a structural model to identify experts' question-answering behavior on the platform. This approach takes a further step in comparison to most previous relevant studies. It identifies the factors lying behind experts' on-platform behavior and their correlations (e.g., Oh, 2012, Guan, et al., 2018) but also incorporates these factors into a pattern that interprets this behavior in terms of actual use (e.g., Lou, et al., 2013; Zhang, et al., 2019).

To address our research objectives, we operationalized the Theory of Planned Behavior (TPB) as a framework that can efficiently interpret experts' perception, intention, and action on an educational CQA platform (Section 2). To establish valid connections between the TPB framework and experts' behaviors, we reviewed the pertinent literature regarding experts' perception of CQA platforms' usability (Section 2.1), question quality (Section 2.2), and added value of answering (Section 2.3). Subsequently, we set up our two research questions (Section 3). Our methodological approach (Section 4) was based on implementing a design study that included developing a questionnaire based on the three dimensions mentioned above (Section 4.1). We shared it with the experts that actively use the 100mentors mobile application (Section 4.2), and we used parallel, factor, and PLS-SEM to analyze the responses (Section 4.3). The results (Section 5) include the research participants' demographics (Section 5.1) and also address the two research questions separately: we present, on the one hand, the factorial analysis of the questionnaire items (Section 5.2) and, on the other hand, the structural model of the emergent factors (Section 5.3). We discuss these results considering our literature review (Section 6). Finally, we summarize our conclusions (Section 7), including potential practical implications (Section 7.1), limitations, and future research directions (Section 7.2).

\section{Literature Review}

As a first step towards a theoretical model, it is important to explore which factors may explain experts' contributions and, more specifically, their question-answering behaviors on a given CQA platform. The TPB (Ajzen, 2015; Cheng, 2019) offers a valid theoretical framework as a basis for interpreting this decision-making process taking place in the minds of the experts. The TPB explains planned behavior as a strong correlation between a person's positive perception of the added value of acting, the intention to act, and the actual behavior (Ajzen, 2015). When applied to the answering process, the TPB suggests that an expert first needs to perceive that a question is worth their while; that it is addressing their relevant expertise (perception of question). When the expert has a positive perception of the question's quality, they might consider answering it (intention to answer). However, before putting intention into action, the expert may want to balance the added value of giving the answer with the cost it would require providing the answer; this can be significantly affected by their personal motives to allocate time and effort on this activity. In addition, the perceived ease of use of the CQA platform, as a proper means for sharing their relevant expertise, might also have an impact on whether they choose to share their answers. We expect that an expert is more likely to answer the question when the added value and ease of use outweighs the cost (the act of answering).

In the light of the TPB, we argue that the factors implicated in experts' question-answering behavior can be described within three different, yet interrelated, dimensions. These dimensions concern experts' perception of a) CQA platforms' usability, b) quality of questions, and c) added value of answering. In the next paragraphs, we operationalize the factors addressing each dimension.

\subsection{Experts' perceived CQA platforms' usability}

The most prominent advantage of CQA platforms is the ease of communication afforded between users (Espina and Figueroa, 2017), as they interact through their asynchronously posted Questions and Answers (Q\&As). The result is the "personalized content produced by means of the collective wisdom of the members of the community" (Figueroa, 2017, pp.11-12). If a CQA platform allows open access, its depository can quickly expand and become a large-scale or even massive knowledge base and thereby draw the attention of information technology researchers. Such examples of particular focus in the related research, include Yahoo! Answers (e.g., Blooma, Goh and Chua, 2012) and Quora (e.g., Wang, et al., 2013). 
The focus of our work, however, is on educational CQA platforms. To more thoroughly investigate them, we used the 100mentors educational CQA platform (web and mobile applications) as our case study. The 100mentors CQA platform drew our attention as its mission includes the improvement of question generation, formulation, and answering within educational/instructional settings. It is an educational technology platform that works with learners from any given learning community, with popularity among K-12 students. Most learners register on the platform at the invitation of an educator or a trainer, who creates the discussion topic on the platform under which these students post their questions. Each topic and each question are considered to be either subject-specific, thematic, or career-related and appears in the question feed of relevant "mentors." They are referred to as "mentors" because they answer both subject- and career-related questions with the company's intent to guide young learners in the long-term. As of March 2021, the 100mentors platform had more than 16,000 registered members (including at least 5,000 mentors), who have posted about 3,500 questions and almost 6,000 answers.

The usability of a CQA platform is the first dimension we investigated to identify factors that influence experts' question-answering behavior. Zooming out, we must recognize that experts who provide answers through CQA platforms are, first and foremost, users of a virtual environment. However, contemporary research on CQA platforms has managed to zoom in, indicating particular elements and features for the usability of these environments (e.g., Nam, Ackerman and Adamic, 2009; Mamykina, et al., 2011; Chua and Balkunje, 2012). Chua and Balkunje (2012, p.211) utilized Nielsen's heuristics (Nielsen, 1994) to elaborate on CQA platform usability in terms of efficiency, effectiveness, and satisfaction. In practice, experts experience these parameters through the website's navigation, organization, and labeling, which are also present in and applicable to mobile applications. Chua and Balkunje (2012, p.212) made a distinction between information sharing (e.g., knowledge creation, external resource sharing, and answer notification) and information organization (e.g., categorization of questions, best answer selection, the establishment of user reputation, and answer period for open questions).

The 100mentors platform is a relatively new entry into the CQA platform landscape; the app under investigation was launched in, 2018. Until now, the 100mentors app has not been analyzed by external academic sources as an educational technology product, although there have been, on numerous occasions, direct feedback surveys of the registered experts (Stokhof, Meli and Lavidas, 2020). Information sharing occurs through bite-sized knowledge creation, as experts record 100-second answer videos, and answer notifications are subsequently pushed to the askers' learning community. Information organization is achieved through (a) question and respective answer tagging on the platform's backend, (b) experts' personalized feed, and (c) visibility of experts' contributions (Chua and Balkunje, 2012).

Although steps have been taken for facilitating the ease with which experts provide answers on the CQA platforms (Nam, Ackerman and Adamic, 2009; Mamykina, et al., 2011; Chua and Balkunje, 2012), the number of shared answers on large-scale CQA platforms is usually inadequate. About a decade ago, Mamykina et al. (2011) argued that general-purpose CQA sites had response rates between $60 \%$ and $90 \%$, except of Stack Overflow (SO) at a rate of $92 \%$. According to Liu \& Jansen (2018), this continues to be the case for three well-known platforms. In their study, they mentioned that response rates were $88.2 \%$ for Yahoo!Answers, 66\% for Naver KnowledgeiN (KiN), and $92.6 \%$ for SO. There is no reason to assume that this would be different in the case of CQA platforms that are not massive. The 100 mentors case confirms this hypothesis. Even though registered experts have viewed almost all their "assigned" questions (and in many cases, repeatedly), many of the questions remain unanswered. Given that these experts are already using a platform as a tool to seamlessly share their knowledge or expertise, they encounter questions that "do not worth their while." Therefore, along with experts' perception of particular features of CQA platforms, we consider that their perception of the questions' quality and the added value of answering are vital for understanding their on-platform behavior and predicting their contribution.

\subsection{Experts' perceived quality of questions on CQA platforms}

The benefits associated with question formulation justify an emphasis on question facilitation on educational CQA platforms, as in 100mentors. Raising questions supports active learning because formulating questions exceeds mere reception of information and requires learners to use higher levels of thinking (AlbergariaAlmeida, 2010). Question formulation also leads to adaptive learning, as self-raised questions derive from learners' existing knowledge structures and personal interests, thus aligning with personal developmental levels and preferences (Newman, 2008). Indeed, questioning supports the development of metacognitive knowledge and skills, as learners must become aware of what they know or do not know, as well as their need to set 
objectives for their inquiry. When these questions receive answers, learners monitor, evaluate, and reflect if and how the answer matches with the information they sought and continue their journey into metacognitive knowledge (Veenman, 2006). Furthermore, the accumulating evidence in recent research suggests that with the proper support, learner questioning is self-motivating, supporting basic psychological needs such as autonomy, competence, and relatedness (Herranen and Aksela, 2019; Ryan and Deci, 2020).

The quality of questions is the second dimension that may influence experts' question-answering behavior (Baltadzhieva and Chrupała, 2015). It raises the question of how we should operationalize question quality. In the present study, the quality of questions was not compared to an objective standard or examined from the learners' perspective. Instead, we focused on the perspective of the experts to find out which question quality factors played a role in their answering behavior. Determining what criteria could be used to measure experts' perception of questions is, nevertheless, complicated. In the literature, relatively little is known about experts' perception of question quality. On the other hand, from the learner's perspective, only three criteria for assessing quality are considered to be significant (Stokhof, 2018): a) the relevance of the question (does it focus on the information needed?), b) the feasibility of the question (is it feasible for the learner to receive an answer?), and c) the learning potential of the question (does the answer contribute to learning progress?).

Relevance seems to be a criterion on how experts assess question quality. Unclear questions are less likely to receive answers, and unclear tagging of questions prevents experts from finding the questions that are relevant to their expertise (Baltadzhieva and Chrupała, 2015). Although "hard to answer" might be a criterion for good questions for some (LaToza and Myers, 2010), most experts might not be willing to answer questions that require too much effort to answer properly (Liu and Jansen, 2018). Similarly, Hao, Shu and Irawan (2014) found that users in SO were actually less likely to answer complex questions. Therefore, the feasibility of the questions is also a criterion that influences experts' response rate. Finally, there are indications that the perceived learning potential also plays a role in question-sharing. Liu and Jansen (2018) found that the informativeness of questions, that is, to what extent they provided potential respondents with sufficient information to understand what was asked, correlated with higher response rates. Hao, Shu and Irawan (2014) reported that users in SO preferred questions that contained a reasonable amount of information, specified the questioner's need, and required opinions from different perspectives. This suggests that the more focused and personalized questions, which offer greater learning potential, are more attractive for experts to answer. By contrast, question features like length or quality were not correlated with response rate (Baltadzhieva and Chrupała, 2015). Moreover, Hao, Shu and Irawan (2014) reported that the overall look and feel of questions did not affect a user's decision to answer. We thus conclude that the criteria of relevance, feasibility, and learning potential seem to mold experts' perception of the questions at hand and, subsequently, affect experts' intention and action to answer. We argue that these criteria are efficient for exploring if and how experts perceive and utilize question quality on an educational CQA platform.

\subsection{Experts' added value of answering on CQA platforms}

Intrinsic and extrinsic motives as the main vehicles toward the added value of answering is the third dimension of investigation for factors that influence experts' question-answering behavior. Several intrinsic motives influence experts' contribution to CQA platforms. Intrinsic motivation derives from one's inherent satisfaction in performing a behavior (Lou, et al., 2013). For experts participating on CQA platforms, this behavior relates to the act of answering questions. According to Jin, Lee and Cheung (2009), experts' satisfaction is the key determinant of continued intention to answer questions. Their satisfaction can derive from the fulfillment of different intrinsic incentives: altruism, self-efficacy, and enjoyment are the most distinctive among them. First, experts' altruism is reflected by their benevolent allocation of time and effort to provide answers, most often without expecting any compensation. Altruism plays a significant role in CQA platforms with a broad range of content (e.g., Nam, Ackerman and Adamic, 2009; Lin and Huang, 2013; Shang, Wu and Li, 2017). Secondly, experts' self-efficacy on CQA platforms relates mostly to experts' self-confidence and self-worth (Nam, Ackerman and Adamic, 2009; Zhang, et al., 2019) which can be positively implicated in their expertise or experience sharing. Last but not least, in terms of intrinsic motives, experts find answering on CQA platforms enjoyable (Oh, 2012; Shang, Wu and Li, 2017) and especially when helping other people (Jin, Lee and Cheung, 2009; Lou, et al., 2013; Fang and Zhang, 2019).

In addition to showing the importance of intrinsic motives for answering questions, the literature also shows that experts may have extrinsic motives for doing so. Extrinsic motivation (or extrinsic/external regulation), in contrast to intrinsic motivation, relates to one's expectation of achieving a favorable outcome (Ryan and Deci, 
2000; Lou, et al., 2013). For experts who actively answer questions on CQA platforms, such favorable outcomes are mostly related to enhancing their reputation and positive social engagement. First, experts' reputations on CQA platforms are, more often than not, directly leveraged through features that are designed exclusively to illustrate users' accomplishments on and off the platform. This usually creates a way to reward experts for their ongoing contributions (Jin, Lee and Cheung, 2009; Lou, et al., 2013). Several gamification features such as sophisticated point systems (Nam, Ackerman and Adamic, 2009; Mamykina, et al., 2011), badges, and up/down votes may also be present (Zaza, Junglas and Armstrong, 2019). Secondly, social engagement refers to a perceived sense of belonging in a community and the establishment of relations with other members of this community (Oh, 2012). Experts may be attracted to a CQA platform due to their interest in its community and their desire to be part of it (Oh, 2012). In addition, one can find the norms of reciprocity that may urge experts to provide answers as part of their social exchange within the community (Oh, 2012; Wu and Korfiatis, 2013; Guan, et al., 2018).

Both intrinsic and extrinsic motives have a remarkable impact on experts' intention to answer questions on CQA platforms, particularly on educational ones. Such incentives appear to flourish somewhere between an experts' perception of question quality and subsequent action toward questions on the platform; in other words, they may play a significant role in their decision to answer.

\section{Research Questions}

Questions can be generated in any context before they reach experts on any given online CQA platform. Therefore, experts are often on the receiving end of questions that are, to a great extent, "out of context" for them. We suggest that these seemingly unmoored questions carry several objective characteristics that account for experts' perception of their quality. Yet, our hypothesis is that an experts' decision to act on a question does not entirely depend on their perception of the questions' objective characteristics. For an expert to progress from perception to action, a clear intention to answer a particular question should be formed. This intention may depend on the experts' intrinsic and extrinsic motives that further explain their attraction toward a particular question, as well as the CQA platform's user-friendliness for contributing a seamless response to a given question. Therefore, our main objective is to identify patterns (in the form of a structural model) that can map experts' perception, intention, and action in answering questions on educational CQA platforms, such as 100mentors.

Our research questions (RQ), therefore, are the following:

RQ1: What are the factors that affect experts' contributions to question-answering platforms?

RQ2: How do these factors interact with each other?

\section{Research Methodology}

\subsection{Research strategy and instrument}

This research followed the strategy of a design study. To pursue our research objectives, a research instrument needed to be developed. First, we developed the theoretical framework and hypothesis for the research instrument, which was informed by the literature review. Then, based on this framework, we formed the items for a pilot questionnaire. Consequently, we tested this pilot questionnaire with a small sample of experts on the 100 mentors platform $(N=9)$. To evaluate the instrument, we conducted qualitative and quantitative analyses. Based on the results of our analyses (Stokhof, Meli and Lavidas, 2020), we revised the pilot questionnaire and developed the final one that was ultimately used for this research. The questionnaire was validated through Exploratory Factor Analysis (EFA, see Section 4.3).

The developed questionnaire investigated the three dimensions defined in our literature review. It included 32 closed-ended items, based on the factors highlighted within each area of interest. The responses to the closedended items were indexed on a 7-point Likert-type scale, with "strongly disagree" at the lowest end (value 1) and "strongly agree" at the highest end (value 7). A response to all close-ended questions was mandatory; therefore, there were no missing values. In addition, the questionnaire included 4 demographic questions (gender, nationality, age, and scientific field of expertise). 


\subsection{Research sample and data collection}

The 100mentors team provided a user list of all 1,151 registered experts ("mentors") that had the mobile application installed on their devices from February 2019 until June 2020; therefore, we consider this to be a convenience sample (Cohen, et al., 2007). In total, 126 experts (more than $10 \%$ of the list) participated in this study.

The questionnaire was set up in a Google Form and the link was sent to potential participants on three different occasions during June 2020. First, they received an email that explained the purpose of the survey and a call-toaction to participate. Then, two weeks later, they received a reminder email with the same link. Finally, they received a final invitation as part of the monthly newsletter sent by the 100 mentors team. In all cases, potential participants were informed that the survey was anonymous, and the collected data would be used for research purposes only.

\subsection{Data analysis strategy}

Initially, we conducted a parallel analysis to determine the structure of the factors of experts' responses in the 32 closed-ended questionnaire items (Horn, 1965). Considering the number of components indicated by the parallel analysis, we conducted EFA using the principal component analysis method to investigate the structure of the instrument. The literature review allowed some hypotheses to be made in advance regarding the factors that the items would form within each dimension, but ultimately the EFA determined the structure of the model (Hair, et al., 2017).

Subsequently, we applied Variance-Based Structural Equation Modeling (VB-SEM), and, more specifically, Partial Least Squares-SEM (PLS-SEM) analysis (Sanchez, 2013; Hair, et al., 2017). PLS-SEM is a contemporary multivariate data analysis method for analyzing relationships between multiple sets of variables (Hair, et al., 2017). This analysis offers flexibility on normality distribution and sample sizes (Hair, et al., 2017). With the PLSSEM analysis we assessed a) the measurement model in terms of its reliability and the validity of its factors and b) the structural model (Hair, et al., 2017). Parallel analysis, factor analysis, and PLS-SEM were all conducted in $R$ (R Core Team, 2018) with the use of the psych (Revelle, 2020) and the plspm package (Sanchez, Trinchera and Russolillo, 2017).

\section{Results}

\subsection{Participants' demographics}

The participants' demographics are presented in Table 1. To identify multivariate outliers, we used Mahalanobis' distance with a Chi-square probability threshold of .001 (Mahalanobis, 1936). Six participants were identified as multivariate outliers; therefore, the final sample size was $N=120$.

Table 1: Participants' demographics $(N=126)$

\begin{tabular}{|l|l|}
\hline Characteristic & Percentage (\%) \\
\hline Gender & \\
\hline Men & 60.3 \\
\hline Women & 38.1 \\
\hline Not disclosed & 1.6 \\
\hline Nationality & \\
\hline Greece & 50 \\
\hline India & 16.7 \\
\hline United Kingdom & 6.3 \\
\hline Germany & 4.8 \\
\hline United States & 2.4 \\
\hline Other & 19.8 \\
\hline Age & \\
\hline$<=26$ & 14.3 \\
\hline $27-35$ & 34.1 \\
\hline $36-44$ & 21.4 \\
\hline $45-53$ & 18.3 \\
\hline$>=54$ & 10.3 \\
\hline Not disclosed & 1.6 \\
\hline & \\
\hline & \\
\hline
\end{tabular}




\begin{tabular}{|l|l|}
\hline Scientific Field $^{\mathbf{a}}$ & \\
\hline Business Management & 50.8 \\
\hline Humanities & 27.8 \\
\hline Economics & 23.8 \\
\hline Physics & 19.0 \\
\hline Computer Science & 14.3 \\
\hline Mathematics & 9.5 \\
\hline Health & 5.6 \\
\hline Other & 13.4 \\
\hline a Each participant can disclose more than one field
\end{tabular}

Considering that 100 mentors is a Greek start-up, it was expected that many experts would be Greeks. The high percentages of experts involved in Business Management were explained due to the 100mentors' strategic focus on this scientific field and the "recruitment" of pertinent experts from well-known companies.

\subsection{Factorial structure of the questionnaire items}

To answer our first research question, we used parallel analysis and, subsequently, exploratory factor analysis. First, to identify the definitive number of factors, we used a principal component analysis method, through which two thousand datasets based on permutations of the raw data were generated; this large number of datasets allowed more precise and reliable results ( $\mathrm{O}^{\prime}$ Connor, 2000). These datasets indicated that five factors had eigenvalues above the corresponding $95^{\text {th }}$ percentile estimates. Secondly, we used the principal component analysis method with oblique rotation to extract the factorial structure of the 32 items for these five factors. Bartlett's Test of Sphericity $(p<0.001)$ indicated that the correlation between the items was adequate for factor analysis. In addition, Kaiser-Meyer-Olkin (KMO=.790) and Measure Sample Adequacy (MSA at least .655) revealed that experts' answers were able to form a satisfactory factorial structure (Field, 2018). However, four items, related to experts' extrinsic motives and question qualities, presented very low loadings $(\lambda<0,4)$ and were excluded to ensure the content validity of the research instrument (Hair, et al., 2017).

Using the same method, factor analysis of the remaining 28 items indicated that the five extracted factors explained 61.55\% of the total variance (Factor 1: 25.43\%; Factor 2: 14.65\%; Factor 3: 9.36\%; Factor 4: 6.53\%; Factor 5: $5.58 \%$ ). Considering the clusters of items that have been formed as a result of our factor analysis, each of the extracted factors was assigned a clear designation: the first factor represented the Perceived Ease of Use $(P E U)$, the second described the Perceived Question Quality (PQQ), the third represented the Expert Intrinsic Motives (EIM), the fourth represented the Expert Extrinsic Motives (EEM), and finally, the fifth represented the Behavioral Intention to Use (BIU). Each of the extracted factors can be clearly appointed to one of the three dimensions that guided the development of the questionnaire: a) PEU and BIU adhere to the CQA platforms' usability, b) PQQ to the quality of questions, and c) EIM and EEM to the added value of answering.

Table 2 presents the measurement model and the items that each factor included. Subsequently, we evaluated the measurement model using PLS-SEM analysis. As Hair et al. (2017) highlighted, all items with loadings between .4 and .7. should be excluded from the measurement model if such an action would lead to an increased convergent validity. Therefore, we excluded four items related to question qualities with loadings close to .5, maintaining the instrument's content validity. A measurement model derived from the 24 remaining items; Cronbach's Alpha $(\alpha)$ and Composite Reliability (CR) (Raykov, 1997) higher than 0.8 indicated a high level of internal consistency reliability for all factors. Moreover, the Average Variance Extracted (AVE), which was higher than 0.5 for each construct, indicated a satisfactory convergent validity (Hair, et al., 2017).

Finally, Table 3 shows Pearson product-moment correlation coefficients and discriminant validity coefficients. The square root of each construct AVE was greater than all correlations among factors (Fornell and Larcker, 1981). Also, Heterotrait - Monotrait (HTMT) ratios between-trait correlations to the within-trait correlations were less than .85 (Henseler, Ringle, and Sarstedt, 2015); therefore, the discriminant validity was considered established. In examining the correlation coefficients among the five factors (see below-diagonal values in Table 3), we found that the BIU has a moderately positive correlation with EIM, the EEM, and the PEU. This finding indicated that the regularity of experts' contributions to the platform was connected to most of the factors. That is to say: experts' intrinsic and extrinsic incentives, in addition to the platform's user-friendliness, could have influenced their answer-recording behavior. Another noteworthy correlation of the same intensity is found between EEM and EIM (.502), confirming that experts' intrinsic and extrinsic motives saw overlap on several 
occasions. All other correlations were also positive but weaker. Of special note are the weak correlations the PQQ had with all factors, which indicates that the perceived quality of questions did not directly affect experts' intention and action. To explore the exact course of interactions between our factors, we constructed their structural model.

Table 2: Descriptive statistics, reliability, and convergent validity indexes of the measurement model

\begin{tabular}{|c|c|c|c|c|c|}
\hline Factors and items & Mean (SD) & $\lambda$ & $\alpha$ & $\mathrm{CR}$ & AVE \\
\hline EEM & $5.89(.79)$ & & .844 & .906 & .760 \\
\hline My answers can have an impact on learners' field knowledge. & & .914 & & & \\
\hline $\begin{array}{l}\text { My answers can have an impact on learners' studies and career } \\
\text { choices. }\end{array}$ & & .845 & & & \\
\hline My answers make me feel helpful or inspiring to learners. & & .855 & & & \\
\hline BIU & $5.55(1.04)$ & & .845 & .897 & .681 \\
\hline I intend to use the 100 mentors app to assist learners. & & .838 & & & \\
\hline I intend to use the 100 mentors app as often as possible. & & .815 & & & \\
\hline I intend to use the 100 mentors app in the future. & & .861 & & & \\
\hline My answers can encourage me to answer more questions. & & .785 & & & \\
\hline PEU & $5.41(1.13)$ & & .849 & .893 & .625 \\
\hline $\begin{array}{l}\text { The } 100 \text { mentors app is clear and understandable for me to } \\
\text { interact with. }\end{array}$ & & .874 & & & \\
\hline It is easy for me to handle my settings on the 100 mentors app. & & .855 & & & \\
\hline $\begin{array}{l}\text { It is easy for me to find help/assistance for the use of the } \\
100 \text { mentors app. }\end{array}$ & & .833 & & & \\
\hline $\begin{array}{l}\text { It is easy for me to select suitable questions on the } 100 \text { mentors } \\
\text { app. }\end{array}$ & & .757 & & & \\
\hline $\begin{array}{l}\text { It is easier for me to answer questions that are in video format } \\
\text { instead of text. }\end{array}$ & & .603 & & & \\
\hline EIM & $5.18(1.05)$ & & .818 & .874 & .581 \\
\hline My answers can offer me enjoyment. & & .724 & & & \\
\hline $\begin{array}{l}\text { My answers can strengthen my confidence in my own expertise } \\
\text { or experience. }\end{array}$ & & .856 & & & \\
\hline My answers can help me become more effective in my job. & & .740 & & & \\
\hline $\begin{array}{l}\text { My answers can help me to become more aware of my } \\
\text { expertise or experience. }\end{array}$ & & .817 & & & \\
\hline My answers can help me expand my social network. & & .658 & & & \\
\hline PQQ & $4.89(1.10)$ & & .847 & .884 & .521 \\
\hline I will only answer questions that are clearly formulated. & & .740 & & & \\
\hline $\begin{array}{l}\text { I will only answer questions that are relevant to my expertise } \\
\text { or experience. }\end{array}$ & & .702 & & & \\
\hline I will only answer questions that I find intriguing. & & .793 & & & \\
\hline I will only answer questions that have a clear focus on my field. & & .705 & & & \\
\hline I will only answer questions that I can explain easily to learners. & & .663 & & & \\
\hline $\begin{array}{l}\text { I will only answer questions that will lead to learners' } \\
\text { understanding of my field. }\end{array}$ & & .746 & & & \\
\hline $\begin{array}{l}\text { I will only answer questions that will enhance learners' curiosity } \\
\text { about my field of expertise. }\end{array}$ & & .698 & & & \\
\hline
\end{tabular}

Table 3: Discriminant validity and Pearson product-moment correlation coefficients

\begin{tabular}{|l|c|c|c|c|c|}
\hline Factors & BIU & PQQ & EEM & EIM & PEU \\
\hline BIU & $(.825)$ & .289 & .532 & .659 & .494 \\
\hline PQQ & .217 & $(.722)$ & .308 & .229 & .292 \\
\hline EEM & .467 & .251 & $(.872)$ & .630 & .256 \\
\hline EIM & .580 & .145 & .502 & $(.762)$ & .378 \\
\hline PEU & .436 & .271 & .287 & .357 & $(.791)$ \\
\hline
\end{tabular}

Notes: Diagonals in parentheses are square roots of the AVE from items. Below-diagonal are correlations among factors and above-diagonal are HTMT ratios of correlations. PEU: Perceived Ease of Use; BIU: Behavioural Intention to Use; PQQ: Perceived Question Quality; EEM: Expert Extrinsic Motives; EIM: Expert Intrinsic Motives 


\subsection{Structural model of factors}

To answer our second research question, we constructed a structural model and tested the significance of the path coefficients (Hair, et al., 2017). We calculated these path coefficients and their $95 \%$ confidence intervals by bootstrapping, a resampling procedure using 5,000 samples with replacement from the original data set (Sanchez, 2013). In 5 out of 10 cases, the direct path coefficients confidence intervals did not include zero. Moreover, the statistically significant path coefficients ranged between a medium and a large effect (Cohen, 1977).

The PQQ did not significantly contribute to direct connections with other factors. The only supported path initiated from the PQQ was the one leading to EEM $(\beta=.250, p<.05)$. EEM, in turn, supported transitions only toward the EIM $(\beta=.497, p<.05)$, which solidified the strong connection between experts' intrinsic and extrinsic motives. Another interesting finding was that EEM did not directly lead to platform-related factors (PEU and BIU). EIM opened two different pathways: one that established a direct connection with the $B I U(\beta=.280, p<.05)$ and one that pointed to the PEU $(\beta=.250, p<.05)$. Moreover, the PEU appeared to have a connection with the BIU $(\beta=.226, p<.05)$, which was an expected outcome since both factors referred to the actual use of the platform. Finally, the four factors (PQQ, EEM, EIM, and PEU) explain $43 \%$ of the BIU total variance.

Based on these results, indicating which were and were not supported paths, we constructed the structural model of factors (Fig. 1). In the scope of the TPB, the PQQ factor reflected experts' initial perception of the questions they might answer. Therefore, it was set as the starting point of the structural model. On the other end of the mode, the BIU factor was linked to experts' platform use regularity in answering questions, and thus to whether they acted on learners' questions. The factors lying between the PQQ and the BIU in the structural model (EEM, EIM, and PEU) reflected experts' intention to progress from the perception of a question to their action on this question. The paths that were not supported and thus were not included in the structural model, illustrated the connections that experts did not systematically make in their perception of, intention toward, and action on the question at hand.

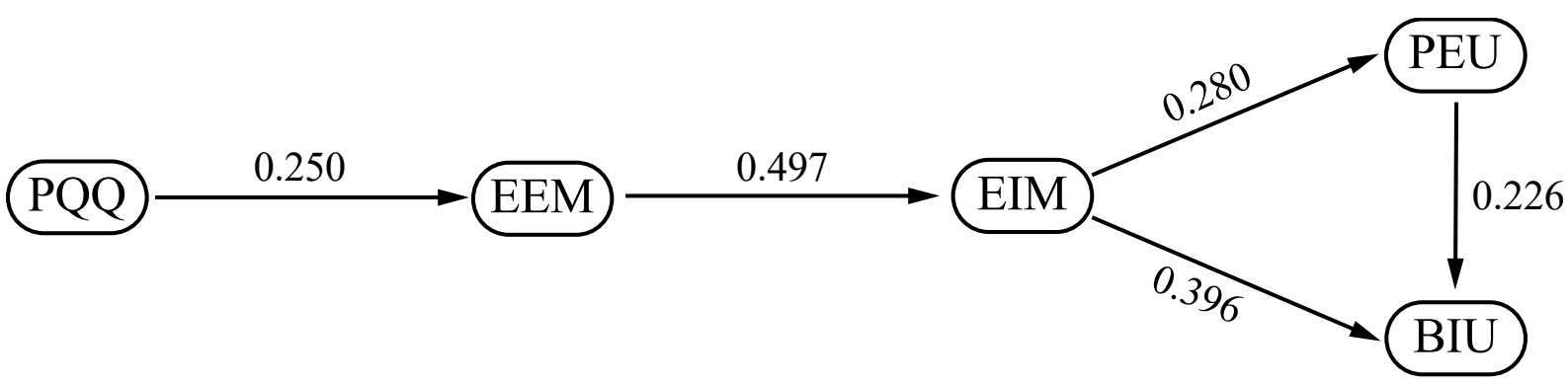

Figure 1: Structural model of factors with path coefficients (supported paths only).

\section{Discussion}

This study explored the factors that influence the regularity of experts' contributions to educational CQA platforms. For this purpose, we used the 100 mentors platform as our case study and analyzed the questionnaire responses that registered experts ("mentors") provided. Based on the pertinent literature, our focus was on exploring experts' perceptions of three distinctive, yet interrelated, fields: CQA platform usability, quality of encountered questions, and added value of answering (intrinsic and extrinsic motives).

We found that five factors can describe experts' behaviors in this context: (a) experts' extrinsic motives, (b) experts' intrinsic motives, (c) the platform's ease of use, (d) experts' behavioral intention to use the platform, and (e) experts' perception of the quality of questions. Most existing relevant studies focus on experts' incentives for sharing their answers on CQA platforms, indicating the added value they find in this activity. Although the distinction between intrinsic and extrinsic motivation is well defined in the literature (e.g., Shang, $\mathrm{Wu}$ and $\mathrm{Li}, 2017$; Ryan and Deci, 2020), we confirmed that there is a strong correlation between these two factors (Lou, et al., 2013), which may explain the lack of consensus on the categorization of the several items suggested in the literature as merely "intrinsic" or "extrinsic" incentives. Concerning the factor of extrinsic motives, which saw the highest degree of agreement, our results confirmed that, by answering, experts intent to make a difference for learners at a personal or educational/vocational level and help other people (Jin, Lee and Cheung, 2009; Lou, et al., 2013; Fang and Zhang, 2019), enjoyment (Oh, 2012; Shang, Wu and Li, 2017), selfefficacy (Nam, Ackerman and Adamic, 2009; Oh, 2012; Lou, et al., 2013; Zhang, et al., 2019), and social network expansion (Oh, 2012). 
CQA platforms' usability is widely addressed in the informational technology framework (e.g., Nam, Ackerman and Adamic, 2009; Shah and Pomerantz, 2010; Mamykina, et al., 2011) and, to a certain degree, can explain experts' initial registration and continued retention on such platforms. The existing literature focuses, to a great extent, on large scale generic (Chua and Balkunje, 2012) and educational CQA platforms, such as SO (Asaduzzaman, et al., 2013), that allow the quantitative analysis of experts' interaction with the several features. Although the 100 mentors platform cannot be directly comparable to the massive platforms, the experts' perceived ease of use confirms previous research's findings. Evidently, the items forming this factor presented rather strong agreement among experts, specifically with regard to items of clear user interaction, assistance availability, and content organization (Nielsen, 1994; Kumar and Goundar, 2019). Concerning the experts' behavioral intention to use the 100mentors app, we found that experts' previous answer-sharing activity worked as a retention motive. However, this item remains controversial for the pertinent research, as some works found its contribution significant (e.g., Burel, et al., 2015), while others report it as indifferent (e.g., Hao, Shu and Irawan, 2014). For the 100mentors' case, we argue that the key to the interpretation of the experts' continued answering, as a behavioral feature, can be found in its positive correlation with the experts' intention to help young learners through the app.

While there is a breadth of research evidence on evaluating answer quality on CQA platforms (e.g., Shah and Pomerantz, 2010), this is not the case for evaluating question quality, for which research remains limited and refers mostly to the questions' formative characteristics, such as textual features and presentation (e.g., Hao, et al., 2014; Calefato, et al., 2015; Liu and Jansen, 2018). However, in the current research, we examined how experts perceive the question quality in terms of content. The items that formed the question quality factor contributed to relatively low loadings and saw a mediocre degree of agreement. This finding supports previous research that shows that the question itself does not determine experts' answering behavior (Asaduzzaman, et al., 2013; Hao, Shu and Irawan, 2014; Calefato, et al., 2015). However, within this factor we found that a question's relevance to experts' expertise or experience was the characteristic they appreciated the most. This result is in agreement with preceding studies (Baltadzhieva and Chrupała, 2015) and explains to a great extent the on-going research for accurate expert-question matching on CQA platforms (Procaci, et al., 2019; Yuan, et al., 2020). This characteristic was also related to the question's clarity (Asaduzzaman, et al., 2013; Hao, Shu and Irawan, 2014; Baltadzhieva and Chrupała, 2015) and challenging nature (Oh, 2012; Procaci, et al., 2019). At the same time, question feasibility and learning potential issues did not concern experts to a significant extent (Hao, Shu and Irawan, 2014; Liu and Jansen, 2018).

To get a further understanding of our results, we constructed a structural model that indicated the ways in which these factors connected. In the pertinent literature, one can find a limited number of similar approaches that address relevant research objectives. Zhang et al. (2018) developed a structural model of factors (individual cognition and community environment), with the operationalization of a knowledge co-creation framework in the social Q\&A community. Lou et al. (2013) utilized the self-determination theory to find correlations between motivation factors and questions quantity/quality. In our study, however, we took a different approach by examining the supported paths under the scope of the TPB (Ajzen, 2015; Cheng, 2019). First, we observed that experts indicated that they perceive a question objectively, implicitly "evaluating" its quality in terms of relevance, feasibility, and learning potential; yet, our findings indicate that experts actually perceived the question quality subjectively, based on its relevance to their own expertise or experience, which guided their further intention and action. Secondly, experts' intention was set mainly by their extrinsic and intrinsic motives, stemming from their aspiration to make a difference for the learner and, to a lesser degree, by the userfriendliness of the platform. Finally, experts' action was linked to their regular use of the platform for answersharing. Our conclusions supported the selection of the TPB as a proper framework for the interpretation of our results in general, and of the structural model in particular. From our point of view, the TPB was proven "allinclusive" in the sense that it holistically described the experiences of experts on educational CQA platforms.

\section{Conclusions}

This study explored experts' behaviors on educational CQA platforms, especially regarding their regular answer contributions. An innovation point, in comparison to the existing literature, is the convergence of three implicated dimensions that, so far, have been examined only separately. Another innovation is the development of the factorial structure for "mapping" experts' interactions with the CQA platforms. At the same time, the current research explores which factors influence experts' online contributions, there is no mention, as far as we know, of how these factors interact with each other. This is particularly important for interpreting and 
predicting experts' behaviors on CQA platforms for both educational and technological purposes (Stokhof, Meli and Lavidas, 2021).

Our results indicated that experts first perceived a question in terms of quality, then their intention to answer was set mainly by their extrinsic-intrinsic motives and the perceived ease of use of the CQA platform, and, finally, their action was linked to the regular use of the platform to share answers. Although these results could be generalizable for both general-purpose and education-specific CQA platforms, they may make more sense for the latter than the former. Since experts were aware that they were responding to learners, who could also be quite young, we posit that they may have made allowances for the questions' quality. Also, they were clearly guided by their desire to have an impact on the learners' subject knowledge and/or career choices; this is exactly what they have signed up for on any given educational CQA platform, such as 100 mentors, which also promotes mentor-like attitudes. In addition, the clarity and ease of interactions with the platform played a role in their intention to use the platform often in the future in order to "assist the learners."

\subsection{Practical implications}

In the light of the TPB, the factorial structure of our analysis indicated that the questions posted on an educational CQA platform are the elements that may or may not trigger an expert to respond. To attract and retain experts on educational CQA platforms, the respective features should be designed in a way that matches their line of perception, intention, and action. Still, we submit that questions are the heart of this process, wherein incentives and platform usability can improve question favorability. To get answers on CQA platforms, learners should not only generate questions that are highly relevant to the topics at hand, feasible for experts to answer, offer learning potential for the asker and/or the community, but also formulate these questions in a way that adheres to experts' thirst for sharing knowledge and experience (e.g., the emergence of potential social impact, direct need for advice/inspiration). To actually get inquiry going, informational technologies should employ educational consulting in order to efficiently bridge the needs of educational CQA platform users, who may be joining the community for similar educational purposes but have dissimilar incentives and concerns.

\subsection{Limitations and future research directions}

This study had some limitations that should be addressed in future research. First, a convenient and small sample was used from the pool of 100 mentors' registered experts; a larger scale of participation in the survey may have resulted in more robust findings. Secondly, the fact that experts were asked to present their personal perception and intention to use a technological system is an issue that usually leads to common method variance (Lavidas and Gialamas, 2019); to minimize this effect, future research with a larger sample can collect data for different factors from different groups of experts (Chang, Van Witteloostuijn and Eden, 2010). Taking the above limitations into account, the next, most urgent future research challenge is the extensive testing of the factorial structural model in large-scale educational CQA platforms and general-purpose CQA platforms.

\section{References}

Ajzen, I., 2015. The theory of planned behaviour is alive and well, and not ready to retire: a commentary on Sniehotta, Presseau, and Araújo-Soares. Health Psychology Review, 9(2), pp. 131-137.

Albergaria-Almeida, P., 2010. Classroom questioning: Teachers' perceptions and practices. In: Procedia - Social and Behavioral Sciences. 2(2), pp. 305-309.

Asaduzzaman, M., Mashiyat, A.S., Roy, C.K. and Schneider, K.A., 2013. Answering questions about unanswered questions of Stack Overflow. In: IEEE International Working Conference on Mining Software Repositories. pp. 97-100. Available at: https://www.cs.usask.ca/ croy/papers/2013/Asaduzzaman MSR2013 SOChallenge.pdf [Accessed 29 August 2021].

Baltadzhieva, A. and Chrupała, G., 2015. Question quality in community question answering forums. ACM SIGKDD Explorations Newsletter, 17(1), pp. 8-13. Available at: https://doi.org/10.1145/2830544.2830547

Blooma, M.J., Goh, D.H.L. and Chua, A.Y.K., 2012. Predictors of high-quality answers. Online Information Review, 36(3), pp. 383-400.

Burel, G., Mulholland, P., He, Y. and Alani, H., 2015. Predicting answering behaviour in online question answering communities. In: HT 2015 - Proceedings of the 26th ACM Conference on Hypertext and Social Media. New York, New York, USA: ACM Press, pp. 201-210. Available at: https://doi.org/10.1145/2700171.2791041

Calefato, F., Lanubile, F., Merolla, M.R. and Novielli, N., 2015. Success factors for effective knowledge sharing in community-based question-answering. In: IFKAD 2015: 10th International Forum On Knowledge Asset Dynamics: Culture, Innovation And Entrepreneurship: Connecting The Knowledge Dots. pp. 1431-1441. Available at: https://collab.di.uniba.it/fabio/wp-content/uploads/sites/5/2014/05/IFKAD_final1.pdf [Accessed 27 August 2021]. 
Chang, S.J., Van Witteloostuijn, A. and Eden, L., 2010. From the editors: Common method variance in international business research. Journal of International Business Studies, 41(2), pp. 178-184. Available at: https://doi.org/10.1057/jibs.2009.88

Cheng, E.W.L., 2019. Choosing between the theory of planned behavior (TPB) and the technology acceptance model (TAM). Educational Technology Research and Development, 67(1), pp. 21-37. Available at: https://doi.org/10.1007/s11423$\underline{018-9598-6}$

Chua, A.Y.K. and Balkunje, R.S., 2012. Comparative evaluation of community question answering websites. In: Chen, H.-H. and Chowdhury, G., eds. 14th International Conference on Asia-Pacific Digital Libraries. Taiwan: Springer, pp. 209218. Available at: https://hdl.handle.net/10356/50815 [Accessed 26 March 2020].

Cohen, J., 1977. Statistical power analysis for the behavioral sciences (revised). New York: Academic Press.

Cohen, L., Manion, L. and Morrison, K., 2007. Research methods in education. 6th ed. London: Routledge Falmer.

Espina, A. and Figueroa, A., 2017. Why was this asked? Automatically recognizing multiple motivations behind community question-answering questions. Expert Systems with Applications, 80, pp. 126-135.

Fang, C. and Zhang, J., 2019. Users' continued participation behavior in social Q\&A communities: A motivation perspective. Computers in Human Behavior, 92, pp. 87-109.

Field, A., 2018. Discovering statistics using IBM SPSS statistics. 5th ed. London: SAGE.

Figueroa, A., 2017. Automatically generating effective search queries directly from community question-answering questions for finding related questions. Expert Systems with Applications, 77, pp. 11-19.

Fornell, C. and Larcker, D.F., 1981. Evaluating structural equation models with unobservable variables and measurement error. Journal of Marketing Research, 18(1), p. 39.

Guan, T., Wang, L., Jin, J. and Song, X., 2018. Knowledge contribution behavior in online Q\&A communities: An empirical investigation. Computers in Human Behavior, 81, pp.137-147. Available at: https://doi.org/10.1016/j.chb.2017.12.023

Hair, J.F., Hult, G.T.M., Ringle, C.M. and Sarstedt, M., 2017. A primer on partial least squares structural equation modeling (PLS-SEM). 2nd ed. Thousand Oaks, CA: Sage.

Hakkarainen, K., 2003. Progressive inquiry in a computer-supported biology class. Journal of Research in Science Teaching, 40(10), pp. 1072-1088. Available at: http://doi.wiley.com/10.1002/tea.10121

Hao, G.K.W., Shu, Z. and Irawan, J., 2014. Good or bad question? A study of programming CQA in Stack Overflow. Nanyang Technological University. Available at: https://www.semanticscholar.org/paper/Good-or-Bad-Question-A-Study-ofProgramming-CQA-in-Hao-Shu/2934af506013d0c4d87f8d555502965260cd2654 [Accessed 12 February 2021]

Henseler, J., Ringle, C.M. and Sarstedt, M., 2015. A new criterion for assessing discriminant validity in variance-based structural equation modeling. Journal of the Academy of Marketing Science, 43(1), pp. 115-135. Available at: https://doi.org/10.1007/s11747-014-0403-8

Herranen, J. and Aksela, M., 2019. Student-question-based inquiry in science education. Studies in Science Education, 55(1), pp. 1-36. Available at: https://doi.org/10.1080/03057267.2019.1658059

Horn, J.L., 1965. A rationale and test for the number of factors in factor analysis. Psychometrika, 30(2), pp. $179-185$. Available at: https://doi.org/10.1007/BF02289447

Jin, X.L., Lee, M.K.O. and Cheung, C.M.K., 2009. Understanding users' continuance intention to answer questions in online question answering communities. In: Proceedings of the International Conference on Electronic Business (ICEB). pp. 679-688.

Kumar, B.A. and Goundar, M.S., 2019. Usability heuristics for mobile learning applications. Education and Information Technologies, 24(2), pp. 1819-1833.

Lakkala, M., 2008. Principles of progressive inquiry. In: Progressive enquiy learning; Paper presented for the inquiry-based learning course, EAHIL 2008. pp. 1-8. Available at: https://wiki.helsinki.fi/download/attachments/41162207/Progressive+inquiry+model introduction.pdf Accessed 1 December 2021]

LaToza, T.D. and Myers, B.A., 2010. Hard-to-answer questions about code. In: Evaluation and Usability of Programming Languages and Tools, PLATEAU'10. New York, New York, USA: ACM Press, pp. 1-6. Available at: https://doi.org/10.1145/1937117.1937125

Lavidas, K. and Gialamas V., 2019. Adaption and psychometric properties of the short forms Marlowe-Crowne social desirability scale with a sample of Greek university students. European Journal of Education Studies, 6(8), pp. 230239.

Le, L.T., Shah, C. and Choi, E., 2017. Bad users or bad content?. In: Proceedings of the 2017 Conference on Conference Human Information Interaction and Retrieval. New York, NY, USA: ACM, pp. 165-174. Available at: https://doi.org/10.1145/3020165.3020181

Lin, F. and Huang, H., 2013. Why people share knowledge in virtual communities?. Internet Research, 23(2), pp. $133-159$. Available at: https://doi.org/10.1108/10662241311313295

Liu, Z. and Jansen, B.J., 2018. Questioner or question: Predicting the response rate in social question and answering on Sina Weibo. Information Processing and Management, 54(2), pp. 159-174. Available at: https://doi.org/10.1016/j.ipm.2017.10.004

Lou, J., Fang, Y., Lim, K.H. and Peng, J.Z., 2013. Contributing high quantity and quality knowledge to online Q\&A communities. Journal of the American Society for Information Science and Technology, 64(2), pp. 356-371. Available at: https://doi.org/10.1002/asi.22750 
Mamykina, L., Manoim, B., Mittal, M., Hripcsak, G. and Hartmann, B., 2011. Design lessons from the fastest Q\&A site in the west. Conference on Human Factors in Computing Systems - Proceedings, 2011, pp. 2857-2866. https://doi.org/10.1145/1978942.1979366

Nam, K.K., Ackerman, M.S. and Adamic, L.A., 2009. Questions in, knowledge in?. In: Proceedings of the 27th international conference on Human factors in computing systems - CHI 09. New York, New York, USA: ACM Press, pp. 779-788. Available at: https://doi.org/10.1145/1518701.1518821

Neshati, M., Fallahnejad, Z. and Beigy, H., 2017. On dynamicity of expert finding in community question answering. Information Processing and Management, 53(5), pp. 1026-1042.

Newman, R.S., 2008. Motivation and self-regulated learning: Theory, research, and applications. In: Schunk, D. H. and Zimmerman, B. J., eds. Motivation and Self-Regulated Learning: Theory, Research, and Applications. Taylor \& Francis Group, pp. 315-337.

Nielsen, J., 1994. Enhancing the explanatory power of usability heuristics. In: Proceedings of the SIGCHI conference on Human Factors in Computing Systems. AMC, pp. 152-158.

O'Connor, B.P., 2000. SPSS and SAS programs for determining the number of components using parallel analysis and Velicer's MAP test. Behavior Research Methods, Instruments, and Computers [online], 32(3), pp. 396-402. Available at: https://doi.org/10.3758/BF03200807

Oh, S., 2012. The characteristics and motivations of health answerers for sharing information, knowledge, and experiences in online environments. Journal of the American Society for Information Science and Technology, 63(3), pp. 543-557. https://doi.org/10.1002/asi.22883

Procaci, T.B., Siqueira, S.W.M., Pereira Nunes, B. and Nurmikko-Fuller, T., 2019. Experts and likely to be closed discussions in question and answer communities: An analytical overview. Computers in Human Behavior, 92, pp. 519-535.

$\mathrm{R}$ Core Team, 2018. R: A language and environment for statistical computing. Vienna, Austria: R Foundation for Statistical Computing. Available at: https://www.r-project.org/ [Accessed 13 March 2021].

Raykov, T., 1997. Estimation of composite reliability for congeneric measures. Applied Psychological Measurement, 21(2), pp. 173-184. https://doi.org/10.1177/01466216970212006

Revelle, W., 2020. Psych: Procedures for psychological, psychometric, and personality research. Evanston. Illinois, USA: Northwestern University.

Ryan, R.M. and Deci, E.L., 2020. Intrinsic and extrinsic motivation from a self-determination theory perspective: Definitions, theory, practices, and future directions. Contemporary Educational Psychology, 61, p. 101860. https://doi.org/10.1016/j.cedpsych.2020.101860

Ryan, R.M. and Deci, E.L., 2000. Intrinsic and Extrinsic Motivations: Classic Definitions and New Directions. Contemporary Educational Psychology, 25(1), pp. 54-67.

Sanchez, G., 2013. PLS path modeling with R. Berkeley: Trowchez Editions. Available at: http://www.gastonsanchez.com/PLS Path Modeling with R.pdf [Accessed 2 December 2021]

Sanchez, G., Trinchera, L. and Russolillo, G., 2017. plspm: Tools for partial least squares path modeling (PLSPM). R package version 0.4.9. Available at: https://cran.r-project.org/package=plspm [Accessed 13 March 2021].

Shah, C. and Pomerantz, J., 2010. Evaluating and predicting answer quality in community QA. SIGIR 2010 Proceedings - 33rd Annual International ACM SIGIR Conference on Research and Development in Information Retrieval. pp. 411-418.

Shang, S.S.C., Wu, Y.-L. and Li, E.Y., 2017. Field effects of social media platforms on information-sharing continuance: Do reach and richness matter?. Information \& Management, 54(2), pp. 241-255. Available at: https://doi.org/10.1016/i.im.2016.06.008

Stokhof, H., de Vries, B., Bastiaens, T. and Martens, R., 2018. Using mind maps to make student questioning effective: Learning outcomes of a principle-based scenario for teacher guidance. Research in Science Education, pp. 1-23.

Stokhof, H., Meli, K. and Lavidas, K., 2021. Exploring factors for experts' response rate on an educational community question-answering platform. In: EARLI 2021 'Education and Citizenship: learning and Instruction and the Shaping of Futures'. Online. Available at:

https://www.researchgate.net/publication/356612051 Exploring factors for experts' response rate on an educa tional Community Question-Answering platform [Accessed 29 November 2021]

Stokhof, H., Meli, K. and Lavidas, K., 2020. To answer, or not to answer, that is the question: Experts' contribution to question-answering platforms. In: ICERI 2020 Proccedings of the 13th International Conference of Education, Research and Innovation. IATED, pp. 2048-2056. Available at: https://library.iated.org/publications/ICERI2020 [accessed 12 January 2021]

Veenman, M.V.J., 2006. Self-questioning as a metacognitive skill. In: Research on questioning. Aveiro: University of Aveiro.

Wang, G., Gill, K., Mohanlal, M., Zheng, H. and Zhao, B.Y., 2013. Wisdom in the social crowd. In: Proceedings of the 22nd international conference on World Wide Web - WWW'13. New York, USA: ACM Press, pp. 1341-1352. https://doi.org/10.1145/2488388.2488506

Wu, P.F. and Korfiatis, N., 2013. You scratch someone's back and we'll scratch yours: Collective reciprocity in social Q\&A communities. Journal of the American Society for Information Science and Technology, 64(10), pp. 2069-2077. https://doi.org/10.1002/asi.22913

Yuan, S., Zhang, Y., Tang, J., Hall, W. and Cabotà, J.B., 2020. Expert finding in community question answering: a review. Artificial Intelligence Review, 53(2), pp. 843-874. 
Zaza, I., Junglas, I. and Armstrong, D., 2019. Studying the artifacts of Q\&A platforms: The central role of the crowd. In: ICIS 2019 Proceedings. p. 18. Available at: https://aisel.aisnet.org/icis2019/crowds social/crowds social/18 [Accessed 21 August 2020].

Zhang, J., Tao, D., Chen, M.H., Sun, Y., Judson, D. and Naqvi, S., 2018. Co-organizing the collective journey of inquiry with idea thread mapper. Journal of the Learning Sciences, 27(3), pp. 390-430.

Zhang, Y., Zhang, M., Luo, N., Wang, Y. and Niu, T., 2019. Understanding the formation mechanism of high-quality knowledge in social question and answer communities: A knowledge co-creation perspective. International Journal of Information Management, 48, pp. 72-84. https://doi.org/10.1016/j.ijinfomgt.2019.01.022

\section{Appendix}

\section{Questionnaire Items}

\section{CQA platform's usability}

1.1) The 100mentors app is clear and understandable for me to interact with.

1.2) It is easy for me to handle my settings on the 100mentors app.

1.3) It is easy for me to find help/assistance for the use of the 100mentors app.

1.4) It is easy for me to select suitable questions on the 100 mentors app.

1.5 ) It is easy for me to answer questions in less than $100 \mathrm{~s}$.

1.6) It is easier for me to answer questions that are in video format instead of text.

1.7) I intend to use the 100 mentors app to assist learners.

1.8) I intend to use the 100 mentors app as often as possible.

1.9) I intend to use the 100mentors app in the future.

1.10) What did we forget to ask you about your user experience within the 100mentors app?

\section{QUESTION QUALITY}

2.1) I will only answer questions that are clearly formulated.

2.2) I will only answer questions that are relevant to my expertise or experience.

2.3) I will only answer questions that I find intriguing.

2.4) I will answer questions that only experts can answer.

2.5) I will only answer questions that have a clear focus on my field.

2.6) I will only answer questions that I can explain easily to learners.

2.7) I will answer a question although its answer can be found in textbooks.

2.8) I will only answer questions that don't require me to research further.

2.9) I will only answer questions that will lead to learners' understanding of my field.

2.10) I will only answer questions that will enhance learners' curiosity about my field of expertise.

2.11) I will only answer questions that I think learners should ask.

2.12) I will answer a question although it has already been answered by another mentor.

2.13) What did we forget to ask you about the questions you find within the 100mentors app?

\section{ADDED VALUE OF ANSWERING}

3.1) My answers can have an impact on learners' field knowledge.

3.2) My answers can have an impact on learners' studies and career choices.

3.3) My answers can help me become more effective in my job.

3.4) My answers can encourage me to answer more questions.

3.5) My answers can provoke other experts to answer.

3.6) My answers can be rewarding through positive feedback.

3.7) My answers can offer me enjoyment.

3.8) My answers can strengthen my confidence in my own expertise or experience.

3.9) My answers make me feel helpful or inspiring to learners.

3.10) My answers can help me to become more aware of my expertise or experience.

3.11) My answers can help me expand my social network.

3.12) What did we forget to ask you about your mentor identity in the 100mentors app?

\section{DEMOGRAPHICS}

I identify my gender as...

My age group is...

The country I currently live in is...

The fields of expertise that I chose for my 100 mentors app feed are... 